\title{
Emerging therapeutic options for sporadic inclusion body myositis
}

\author{
This article was published in the following Dove Press journal: \\ Therapeutics and Clinical Risk Management \\ 25 September 2015 \\ Number of times this article has been viewed
}

\section{Lindsay N Alfano \\ Linda $\mathrm{P}$ Lowes}

Nationwide Children's Hospital, Center for Gene Therapy, Columbus, $\mathrm{OH}, \mathrm{USA}$
Correspondence: Lindsay N Alfano Nationwide Children's Hospital, Center for Gene Therapy, 700 Children's Dr, Suite OCIA, Room ABI042, Columbus, $\mathrm{OH} 43205$, USA

$\mathrm{Tel}+\mathrm{I} 614722688$ I

Fax + I 6/47226878

Email lindsay.alfano@nationwidechildrens. org

\begin{abstract}
Sporadic inclusion body myositis is the most common inflammatory muscle disorder preferentially affecting males over the age of 40 years. Progressive muscle weakness of the finger flexors and quadriceps muscles results in loss of independence with activities of daily living and eventual wheelchair dependence. Initial signs of disease are often overlooked and can lead to mis- or delayed diagnosis. The underlying cause of disease is unknown, and disease progression appears refractory to available treatment options. This review discusses the clinical presentation of inclusion body myositis and the current efforts in diagnosis, and focuses on the current state of research for both nonpharmacological and pharmacological treatment options for this patient group.
\end{abstract}

Keywords: myositis, inclusion body myositis, inflammatory myopathy, treatment, function, outcomes

\section{Introduction}

Sporadic inclusion body myositis (IBM) is one of a group of inflammatory muscle disorders resulting in progressive muscle weakness. It is the most common inflammatory disorder preferentially affecting men (2:1 over females) over the age of 40-50 years. ${ }^{1-3}$ Incidence and prevalence of IBM have not been well characterized. A recent review estimates incidence of IBM between 0.9 and 3.2 per million per year; however, this varied dramatically based on geographic location. ${ }^{4-6}$ Reported prevalence also widely varies between 0.3 and 13.9 per 100,000 , and again varied by geographical location..$^{5}$ The underlying cause of IBM is not yet fully understood and has been hypothesized to involve protein dysregulation, mitochondrial dysfunction, autoimmune factors, myonuclear degeneration, and impaired nucleic acid metabolism. ${ }^{7-14}$ Despite the continued investigation into the cause of IBM, histopathological characteristics of muscle have been traditionally used in confirming the diagnosis. The essential physical features for a diagnosis of IBM include initial muscle weakness presenting in a characteristic pattern of quadriceps and finger flexors in individuals over the age of 40 years. Griggs criteria was first developed and adopted to standardize diagnosis of this disease, followed by the Medical Research Council (MRC) criteria, and more recently the European Neuro Muscular Centre (ENMC) IBM research diagnostic criteria $2011 .^{15-17}$ Griggs criteria defines IBM when rimmed vacuoles and inflammatory infiltrates are present; however, inconclusive biopsy results were often found in patients fitting the clinical presentation of IBM and refractory to any treatment. The MRC and ENMC criteria were developed to enhance ease of diagnosis when histopathological features were not found in muscle biopsies, hypothesized to potentially occur earlier in disease presentation. ${ }^{16,17}$ As treatments become available, the potential for earlier 
diagnosis has implications for earlier onset of treatment during a period when muscles may be more responsive.

There is currently no successful treatment to stop or delay the progression of disease in IBM. In the absence of a conclusive muscle biopsy, often IBM was differentiated from polymyositis or dermatomyositis by being refractory to immunosuppressive therapies. ${ }^{18-20}$ Current research is focused on identifying the fundamental causes of disease progression, as well as initial clinical trials aimed at reducing disease burden or treating symptoms of the disease.

\section{Clinical description}

IBM is characterized by progressive, often asymmetric, muscle weakness preferentially affecting skeletal muscle. The two muscle groups often initially affected are the quadriceps and finger flexors. The slowly progressive nature of the disease frequently leads to delayed diagnosis (discussed further), as both patients and clinicians attribute slight changes in function to the aging process. ${ }^{21}$ Due to the initial quadriceps weakness, patients often report difficulty with stairs or falls as the first signs of the disease. ${ }^{1}$ Later in the disease, ankle dorsiflexion and elbow extension weakness frequently develop, increasing susceptibility to falls and the difficulty of transfers, such as rising from a chair.

As the disease progresses, weakness expands throughout the upper and lower limbs, leading to eventual loss of independent ambulation and wheelchair use. Additionally, weakness of the distal, then proximal, muscles of the upper extremities quickly leads to loss of independence with activities of daily living, such as grooming, dressing, and hygiene activities. ${ }^{22,23}$ The rate of disease progression can vary widely, with some reports linking increased severity associated to a later age of onset. ${ }^{3,24,25}$

Several natural history studies have reported declines in quadriceps strength ranging between $12.5 \%$ and $27.9 \%$ over 1 year. ${ }^{3,25-28}$ Reports of composite quantitative muscle testing in patients with IBM appear to decline $9.2 \%$ in 1 year or $4 \%$ in 6 months. ${ }^{27-29}$ In a sample of 85 ambulatory individuals with IBM, quadriceps strength was computed as a percentage of age, sex, and height predictions. The deficits in this group ranged from $85 \%$ of predicted strength to less than $1 \%{ }^{28}$ While these trends are useful in clinical trials, it is important for the clinician to remember that changes in strength and function are often not linear and that rates of decline or stability may change across the disease. ${ }^{25,27}$

The change in function and progressive disability has also been described. The mean or median loss of independent ambulation ranged between 7 and 14 years from the date of diagnosis in different cohorts, with an average change in distance walked on the 6-minute walk test (6MWT) decreasing $34 \%$ over a 4 -year period of time. ${ }^{3,24,27}$ The IBM functional rating scale was designed as a measure of disease severity and rates the difficulty to complete ten items related to swallowing, fine motor, and gross motor tasks. ${ }^{30}$ Varying rates of decline using this outcome have been reported, ranging from $22.3 \%$ over 4 years to $13.8 \%$ over 1 year. ${ }^{3,27}$

In a subset of patients with IBM, insidious onset dysphagia creates swallowing difficulties and choking. ${ }^{1}$ As with the initial muscle wasting, early signs of dysphagia can be overlooked as aging related choking or coughing associated with eating or drinking. In $40 \%-50 \%$ of patients, however, dysphagia becomes quite debilitating later in disease progression. ${ }^{31,32}$ Interestingly, although IBM is more common in men, there is some evidence suggesting that dysphagia is more commonly the initial presenting symptom in women. ${ }^{33}$

Cardiac function appears to be spared in IBM, although case studies have been published reporting various cardiomyopathies coexisting in patients with IBM. ${ }^{34-37}$ While heart function may be preserved, there are reports of sleep disordered breathing being identified primarily later in disease progression, although not necessarily correlated to severity of peripheral muscle weakness. ${ }^{38,39}$ Respiratory decline has also been reported as the most common cause of death in a long-term follow-up of patients with IBM. ${ }^{22}$

\section{Diagnosis and markers of disease}

Many patients with IBM report a difficulty finding the correct diagnosis. This is likely related to the rarity of this disease in combination with the slowly progressive changes often attributed to aging. The mean time from symptom onset to correct diagnosis ranges in published work between 3 and 5 years, with many patients receiving at least one incorrect diagnosis prior to the diagnosis of IBM..$^{21,28,40,41}$ As described earlier there are multiple diagnostic criteria that have been developed to standardize diagnosis of IBM. Despite the wide use of these criteria among neuromuscular professionals, a recent study found that all criteria evaluated had high specificity (ranging 98\%-100\%), but they had wide-ranging sensitivities $(11 \%-84 \%)$ with the potential of several false negatives or missing a diagnosis of IBM when present. ${ }^{42}$

Traditionally, muscle biopsies have been used to confirm the diagnosis of IBM, requiring a combination of vacuolated muscle fibers, inflammatory myopathy with mononuclear cell invasion, amyloid accumulation, or $15-18 \mathrm{~nm}$ filaments. ${ }^{15-17}$ Often, a combination of clinical and pathological features is 
required to make the diagnosis in the presence of a nondefinitive biopsy. Many factors may contribute to the difficulty in observing hallmark pathological features in a biopsy, such as those related to biopsy site, distribution of pathology, or the effect of disease progression on distribution or occurrence of pathology. ${ }^{16,21,43}$

In the continued pursuit to better understand the disease pathogenesis of IBM, several biomarkers have been evaluated for both their role in the disease process and as a potential for use in differentiating IBM from other inflammatory myopathies. Due to the presence of protein aggregation, inflammation, and cell death in muscle biopsy of patients with IBM, biomarkers related to inflammation, autophagy, and protein aggregation have been proposed, such as tau, $\beta$-amyloid, Clathrin, and Beclin I. While these have been specifically reported, others recommend targeting markers of general protein aggregation, such as LC3 and p62, as no one protein is consistently found in muscle cells of patients. ${ }^{44}{ }^{49}$ Recently, autoantibodies have been investigated as a serological marker for IBM, with autoantibodies against cytoplasmic 5 ' nucleotidase showing promise as they demonstrate moderate sensitivity (49\%-76\%) and high specificity (91\%-96\%) for IBM. ${ }^{10,50-53}$ However, further research is needed as this marker was detected in other patients with other inflammatory myopathies or autoimmune disorders, so is not sensitive to IBM. ${ }^{10}$

As techniques for genetic analysis continue to advance, new findings relating genes and polymorphisms to disease susceptibility or severity of disease progression are being investigated. Changes in HLA-DRB1, HLA-DRB3, HLADRA, and BTNL2 have been associated with susceptibility of developing IBM, with HLA-DRB1, HLA-DRB3, HLADRA, and BTNL2 being reported with increased susceptibility to IBM and HLA-DRB1 increasing risk up to ten times in a Western Australian population. ${ }^{54-57}$ Alternatively, HLA-DRB loci DRB4 and DRB5 and a polymorphism in the TOMM40 gene appear to have a protective effect in reducing risk of IBM. ${ }^{58}$ Additionally, allele changes in the TOMM40 gene were also associated with later onset of symptoms, up to 4 years; and when these changes were combined in carriers of the APOE genotype $\varepsilon 3 / \varepsilon 3$, onset was delayed 5 years. ${ }^{59}$

Additionally, use of MRI to assist or confirm diagnosis of IBM has been investigated. ${ }^{60-63}$ Fatty infiltration compared to inflammation was a hallmark of IBM compared to other inflammatory myopathies such as polymyositis. ${ }^{61,63}$ Muscles of the thigh, including vastus intermedius and medialis, were characteristically affected, while the rectus femoris and posterior compartment of the thigh were often spared or less involved. ${ }^{60,62,63}$ The medial gastrocnemius was frequently involved with inconsistent involvement of the finger flexors, posterior tibialis, and soleus muscles. ${ }^{60,62,63} \mathrm{~A}$ blinded assessment of MRI scans in 137 patients with myopathies (17 with definite IBM) reported the ability to detect definite IBM of the typical pattern with $59 \%$ sensitivity and $100 \%$ specificity. ${ }^{60}$ Sensitivity improved when considering the diagnosis of IBM for both typical and consistent patterns (sensitivity of 94\%). Authors reported similar accuracy for patients both early and late in disease progression. ${ }^{60}$

\section{Treatment options Nonpharmacologic treatment options} Exercise

Historically, exercise has been recommended cautiously to avoid the effect of overwork leading to more rapid muscle breakdown; however, recent research is pushing the field to think of exercise differently. Although, there have been no large randomized-controlled trials evaluating the effect of exercise in IBM to date, several small studies have been published reporting safety of a low-to-moderate resistance program. ${ }^{2,31-33,64}$ These studies were limited by small sample sizes and relatively short follow-up periods. While the study completed by Arnardottir reported no improvement in strength or function after the home program, they reported a benefit to using exercise to prevent loss of muscle strength over time.$^{65}$ Separate cohorts of studies (ranging from case studies to seven patients enrolled) investigating possible exercise regimes in IBM reported improvements in strength, function, and aerobic capacity., ${ }^{2,32,33,64}$ While additional research is needed in a larger randomized cohort, initial results from small studies indicate that exercise appears safe and may produce the benefit of mild increases or maintenance of strength and function. In a disease that has appeared refractory to all treatment options, these findings suggest a possible tangible treatment for patients to temporarily reduce the progression of IBM over time. Implementation of any exercise regimen should be supervised by a trained physical therapist to ensure safety in an aging population with unique patterns of muscle weakness.

\section{Orthoses}

The presenting quadriceps weakness is often accompanied by anterior tibialis muscle weakness leading to progressive foot drop. Falls frequently occur in this population due to knee collapse or tripping due to foot drop or uneven surfaces. There have not been any large studies investigating use of orthoses in IBM, although bracing has the potential 
to reduce fall occurrence in this cohort. Bernhardt evaluated use of a specific stance control orthosis unilaterally in a small cohort of patients with IBM. The braces were welltolerated; however, they resulted in slower walking velocity and cadence, which may not be preferable for all patients. ${ }^{49}$ Patients with less weakness and those wearing the orthosis more frequently reported more stability and fewer falls. Further research is needed into the optimal orthotic intervention in this population, which is likely to be individualized to specific patient needs. A lightweight orthosis providing additional stability for the quadriceps muscles, such as one with anterior shell support, may also assist in reducing occurrence of foot drop and falls.

\section{Pharmacologic intervention}

There is no current treatment effective at halting or slowing disease progression in IBM. However, several treatments aimed at improving symptoms of IBM have been studied. Most research has focused on repurposing existing medications for use in patients with IBM, although development of novel compounds and therapies has begun in recent years (Figure 1).

\section{Immunosuppressants}

Due to the presence of muscle inflammation in patients with IBM, a variety of immunosuppressants have been trialed in IBM cohorts. While one case study reported a sustained muscle strength improvement in a patient with IBM and antiJo-1 antibodies, larger studies have been inconclusive or have shown a decrement in muscle strength during prednisone treatment. ${ }^{66,67}$ Various combinations of prednisone with other compounds have also been trialed with mixed results, leading to negative consensus in the field for use of these compounds in IBM. Pilot studies of prednisone and mycophenylate, cyclosporin-A, or tacrolimus indicated potential for treatment; however, larger trials reported no long-term positive effect in IBM. ${ }^{68,69}$ Likewise, a trend toward improvement in dysphagia associated with IBM was reported in a trial of intravenous immunoglobulin (IVIg) and a small case series combining IVIg and prednisone, ${ }^{70,71}$ however, these changes have not been reported in the long-term in a larger cohort. ${ }^{72,73}$ Studies of IVIg in larger cohorts have reported inconclusive results, including minor strength gains in some muscles of the leg with the strength of other muscles declining over the same period..$^{71,74}$ Additionally, methotrexate has been evaluated in IBM. However, a double-blind trial of methotrexate versus placebo reported no difference in disease progression measured by muscle strength over 48 weeks. ${ }^{75}$ Additionally, there are reports of a connection between immunosuppressive therapies and potential disease exacerbation. ${ }^{24,76}$

Although small and unblinded trials have reported trends toward or improvement in few patients, these results have not been replicated in large-randomized controlled trials. While immunosuppressive therapy may have a potential for benefit in a small subset of patients, the characteristics of such a cohort have not been well defined. Current literature does not support the use of immunosuppressive therapy to maintain or improve muscle strength in IBM.

\section{Tumor necrosis factor blocking agents}

Tumor necrosis factor (TNF) blocking agents, etanercept and infliximab, have been evaluated in IBM, without dramatic improvements in disease progression. A small study comparing etanercept and two separate control groups (a natural

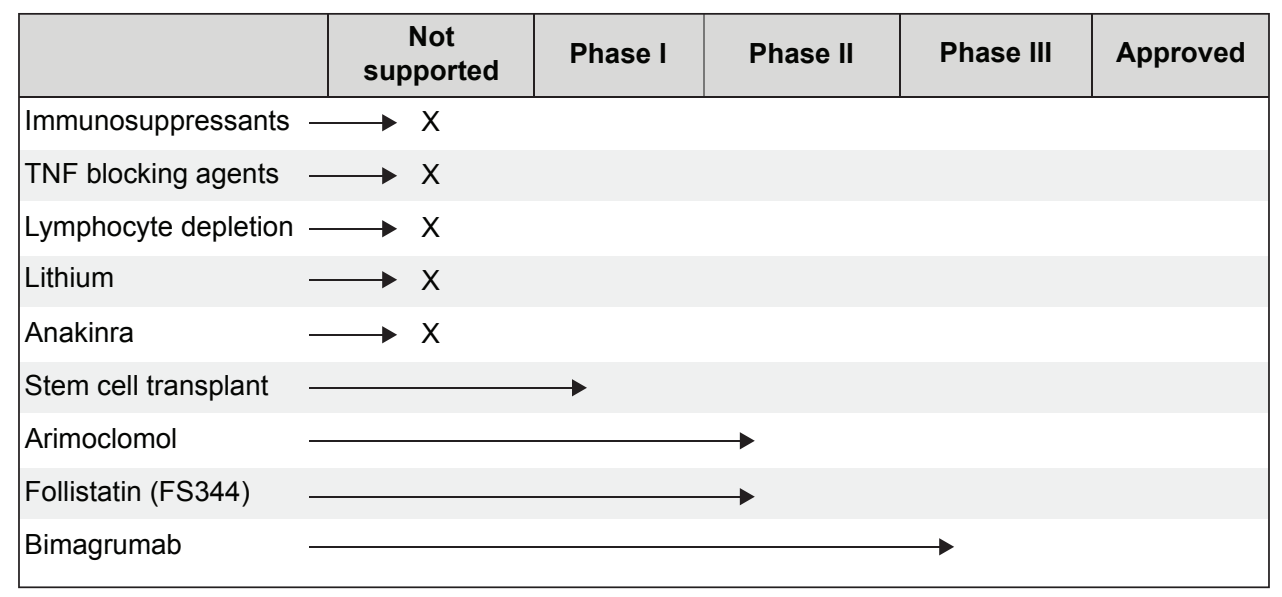

Figure I Progress of drug development and testing in IBM on a scale from not currently supported or recommended based on best available evidence to full approval. Abbreviations: TNF, tumor necrosis factor; IBM, inclusion body myositis; X, not supported by current evidence, no current studies underway. 
history group and a placebo group from another trial) found no difference in strength at 6 months, but a slight improvement in the hand grip strength of the treated group at 12 months. ${ }^{77}$ The clinical significance of this finding is unknown.

Infliximab was also tested in a cohort of patients with refractory myositis, including four patients with IBM. Treatment side effects were significant and resulted in drop out of $25 \%$ of the cohort. Additionally, no change in muscle strength was measured, and $23 \%$ of the cohort demonstrated increased muscle inflammation on MRI posttreatment. Thus, infliximab is not considered an alternative treatment option in patients with refractory myositis. ${ }^{78}$ TNF blocking agents are likely not a viable treatment option as early trial results point to limited benefit which may not provide enough improvement to outweigh potential treatment risks and cost.

\section{Lymphocyte depletion therapy}

Alemtuzumab is a compound targeted against CD52, a glycoprotein expressed on mature T-cells. It has been proposed that use of this compound in IBM has the potential to reduce muscle weakness by decreasing muscle injury related to T-cell response. Results from a pilot trial of 13 patients receiving one course of alemtuzumab treatment lasting 4 days were promising. ${ }^{79}$ Authors reported differential gain in strength at 6 months compared to the previous 12-month observation, and 4 of 13 showed an improvement in strength and 6 reported improved activities of daily living. ${ }^{79}$ Concerns have been raised with this study design and the reported conclusions, recommending further analysis of this compound and evaluation in a blinded trial. ${ }^{80}$ A case study of rituximab in a patient with comorbid rheumatoid arthritis resulted in muscle wasting and weakness of quadriceps muscles following prednisone treatment. Rituximab improved symptoms related to the rheumatoid arthritis leading to remission, but had no effect on muscle strength. ${ }^{76}$

\section{Other treatments}

Lithium was proposed as a potential treatment in early preclinical studies. Muscles of patients with IBM are characterized by protein aggregates containing amyloid- $\beta$ as well as phosphorylated tau. Early in vitro and mice studies reported reduced amyloid- $\beta$ precursor protein levels and improved proteasome function indicating a potential benefit for patients with IBM. ${ }^{81,82}$ However, a 12-month study enrolling 15 participants produced no significant benefit in muscle strength. ${ }^{83}$

Anakinra, an interleukin-1 receptor antagonist, was evaluated in patients with IBM with the goal of reducing amyloid deposits and improving muscle strength and function by blocking IL-1 $\beta$. An open-label pilot study in four patients resulted in no significant improvement or stabilization in hand grip strength or muscle strength measured by manual muscle testing. ${ }^{84}$ In a separate study of 15 patients with refractory myositis (five with IBM) receiving daily self-administered injection of anakinra for 12 months, results were mixed. ${ }^{85}$ Seven patients demonstrated improvement across the 12-month study period on the International Myositis Assessment and Clinical Studies group global assessment, with one patient with IBM showing improvement after 3 months. Three patients with IBM did not demonstrate improvement, and one withdrew from the study due to worsening of symptoms. ${ }^{85}$ Further research is needed to determine efficacy in a larger cohort or to identify the specific characteristics of a potential subgroup of responders to this treatment.

Early work in stem cell transplantation is being evaluated for patients with IBM. Mesoangioblasts from muscle of patients with IBM display defective terminal differentiation with absence of MyoD mRNA expression in myotubes. This block can be corrected in vitro by transient MyoD transfection. ${ }^{86,87}$ A Phase I clinical trial is underway (expected enrollment of ten patients) and is designed to evaluate the effect of high dose cyclophosphamide and antithymocyte globulin with hematopoetic stem cells on progression of myositis. ${ }^{88}$ Results are anticipated in 2017.

Arimoclomol induces increased production of heat shock protein, which is typically increased in vivo in response to cellular stress. A 4-month study in 24 patients with IBM was conducted to evaluate the safety and potential to increase muscle strength in this disease. ${ }^{89}$ Initial reports indicate that arimoclomol was safe and could have a potential for treatment in IBM. ${ }^{90}$ Further research is needed in a larger cohort to confirm these preliminary results.

Follistatin, a myostatin inhibitor, has the potential to increase muscle mass. A Phase I/IIa study is currently underway in patients with IBM. ${ }^{91}$ The alternatively spliced isoform of follistatin (FS344) is injected into the quadriceps muscles of nine patients with IBM to evaluate safety and initial signs of efficacy. Results from a Phase I/IIa study in Becker muscular dystrophy show promising results, but have not yet been reported in the IBM cohort. ${ }^{92}$

Bimagrumab acts to inhibit activin type II receptors in the myostatin pathway to potentially increase muscle size in patients with IBM. ${ }^{93}$ Initial results of a safety study of this compound in eleven subjects with IBM receiving one dose of bimagrumab compared to three receiving placebo indicated that the compound was well-tolerated with 
significant improvements in muscle volume and lean body mass. ${ }^{94}$ A 16-week observation phase in 12 subjects described a significant improvement in distance walked on the 6MWT peaking at 16 weeks. ${ }^{94}$ A pivotal trial in 240 subjects with IBM is currently ongoing to evaluate changes in the distance walked on the $6 \mathrm{MWT}$ after 52 weeks. ${ }^{95}$

\section{Efficacy outcomes}

Selection of appropriate outcome measures, whether clinically or as part of research trials, is often overlooked or undervalued. Clinically, patients often ask how their disease progression relates to the natural history of the disease. Longitudinal natural history trials provide access to the general trend of disease progression in patients with IBM; however, these may be less meaningful to an individual patient. These are no substitutes for a thorough evaluation of disease progression involving imaging, quantitative strength testing, as well as performance of functional tests, including walking, stair climbing, and balance tasks, during regularly scheduled clinic visits. Discussing an individual's trajectory can help clinicians manage a patient's expectations for the future in the presence of an advancing, progressive disease.

To effectively measure efficacy as part of clinical trials, outcome measure selection is crucial. This is particularly important in a slowly progressive disease such as IBM. Early clinical trials often attempt to measure differences in strength or function across groups in short-term trials, typically 48 weeks or less. In a disease like IBM, where initial ability may widely vary and where natural history studies report a range of disease progression, improper or careless choice of outcome may result in false negative outcomes. While the US Food and Drug Administration prefers use of a clinically meaningful, typically functional, outcome measure for product approval, guidelines for surrogate measures have been reported. ${ }^{85}$ Where traditionally, an outcome such as timed walking would be chosen as the primary efficacy outcome for clinical trials, a surrogate measure such as strength or imaging may be a suitable alternative for short-term trials of innovative products. ${ }^{28}$

Another important point to contemplate when selecting outcomes for clinical trials is the overall burden of testing on an individual throughout the course of a visit or the study. Often, clinical evaluation of a subject lasts between 1 and 3 hours for any given visit. In a disease such as IBM, with significant weakness often resulting in increased frequency of falls, the level of fatigue caused by assessments should be considered. For example, while the 6MWT has been successfully implemented in clinical trials involving products eventually achieving registration, it is important to evaluate all options available if a timed walking test is being chosen as a primary efficacy outcome. In IBM in particular, patients tend to walk at the same speed, which is often the fastest speed that minimizes falls. ${ }^{96}$ Choice of the 2-minute walk test, included in the National Institutes of Health toolbox, may be a suitable alternative to capture the same information (distance walked on a timed test) while reducing the overall burden of testing.

Finally, a perfectly designed study with optimal safety and efficacy outcome selected has the potential to be derailed by variability in patient performance and clinical evaluator ratings. Reducing the burden of testing can minimize some variability of subject performance. However, more important is ensuring that subject motivation is considered. While it is near impossible to guarantee consistent motivation of a subject across visits or study sites, understanding and attempting to minimize the effect of motivation on subject performance will likely reduce variability associated with an individual's performance.

Additionally, establishing clinical evaluator intra- and interrater reliability can assist in reducing variability of trial data. The more subjective an outcome measure, the more likely reliability issues are to arise. Establishing intra- and interrater reliability values on the outcomes included in a trial may assist in post hoc statistical analysis of study results. The process of determining evaluator reliability may in itself reduce some variability across sites in a multisite trial.

\section{Conclusion}

IBM is a slowly progressive disease with currently no effective pharmacologic treatment to slow or halt the disease process. While the underlying cause has yet to be determined, many trials are underway to treat major symptoms of the disease. The success of these trials is dependent on selection of appropriate outcome measures and proper clinical evaluator training. Implementation of an individualized exercise regimen or orthotic intervention may provide patients with a tangible option to temporarily reduce the progression of disease or reduce falls, although further research is needed to determine the full effects of these treatment options.

\section{Disclosure}

Dr Alfano currently serves as a clinical evaluator for the ongoing follistatin trial in IBM at the Research Institute at Nationwide Children's Hospital. Dr Lowes also serves as a clinical evaluator for the ongoing clinical trial of the follistatin compound in IBM at The Research Institute at Nationwide Children's Hospital. She also receives funding 
from Novartis Pharmaceuticals as principal investigator of a longitudinal natural history study in IBM. The authors report no other conflicts of interest in this work.

\section{References}

1. Paltiel AD, Ingvarsson E, Lee DK, et al. Demographic and clinical features of inclusion body myositis in north America. Muscle Nerve. Epub December 30, 2014.

2. Flachenecker P. Epidemiology of neuroimmunological diseases. J Neurol. 2006;253(Suppl 5):V2-V8.

3. Cortese A, Machado P, Morrow J, et al. Longitudinal observational study of sporadic inclusion body myositis: implications for clinical trials. Neuromuscul Disord. 2013;23:404-412.

4. Lynn SJ, Sawyers SM, Moller PW, O’Donnell JL, Chapman PT. Adult-onset inflammatory myopathy: North Canterbury experience 1989-2001. Intern Med J. 2005;35(3):170-173.

5. Meyer A, Meyer N, Schaeffer M, Gottenberg JE, Geny B, Sibilia J. Incidence and prevalence of inflammatory myopathies: a systematic review. Rheumatology (Oxford). 2015;54(1):50-63.

6. Wilson FC, Ytterberg SR, St Sauver JL, Reed AM. Epidemiology of sporadic inclusion body myositis and polymyositis in Olmsted County, Minnesota. J Rheumatol. 2008;35(3):445-447.

7. Greenberg SA. Biomarkers of inclusion body myositis. Curr Opin Rheumatol. 2013;25(6):753-762.

8. Herbert MK, Stammen-Vogelzangs J, Verbeek MM, et al. Disease specificity of autoantibodies to cytosolic $5^{\prime}$-nucleotidase $1 \mathrm{~A}$ in sporadic inclusion body myositis versus known autoimmune diseases. Ann Rheum Dis. Epub February 24, 2015.

9. Joshi PR, Vetterke M, Hauburger A, Tacik P, Stoltenburg G, Hanisch F. Functional relevance of mitochondrial abnormalities in sporadic inclusion body myositis. J Clin Neurosci. 2014;21:1959-1963.

10. Lloyd TE, Christopher-Stine L, Pinal-Fernandez I, et al. Cytosolic $5^{\prime}$-nucleotidase $1 \mathrm{~A}$ is a common target of circulating autoantibodies in several autoimmune diseases. Arthritis Care Res. Epub April 17, 2015.

11. Morosetti R, Gliubizzi C, Sancricca C, et al. TWEAK in inclusionbody myositis muscle: possible pathogenic role of a cytokine inhibiting myogenesis. Am J Pathol. 2012;180:1603-1613.

12. Moussa CE, Fu Q, Kumar P, et al. Transgenic expression of $\beta$-APP in fast-twitch skeletal muscle leads to calcium dyshomeostasis and IBMlike pathology. FASEB J. 2006;20(12):2165-2167.

13. Pinkus JL, Amato AA, Taylor JP, Greenberg SA. Abnormal distribution of heterogeneous nuclear ribonucleoproteins in sporadic inclusion body myositis. Neuromuscul Disord. 2014;24:611-616.

14. Rygiel KA, Miller J, Grady JP, Rocha MC, Taylor RW, Turnbull DM. Mitochondrial and inflammatory changes in sporadic inclusion body myositis. Neuropathol Appl Neurobiol. 2015;41(3):288-303.

15. Griggs RC, Askanas V, DiMauro S, et al. Inclusion body myositis and myopathies. Ann Neurol. 1995;38:705-713.

16. Hilton-Jones D, Miller A, Parton M, Holton J, Sewry C, Hanna MG. Inclusion body myositis: MRC Centre for Neuromuscular Diseases, IBM workshop, London, 13 June 2008. Neuromuscul Disord. 2010;20:142-147.

17. Rose MR, Group EIW. 188th ENMC International workshop: inclusion body myositis, 2-4 December 2011, Naarden, The Netherlands. Neuromuscul Disord. 2013;23:1044-1055.

18. Lazarou IN, Guerne PA. Classification, diagnosis, and management of idiopathic inflammatory myopathies. J Rheumatol. 2013;40:550-564.

19. Machado PM, Dimachkie MM, Barohn RJ. Sporadic inclusion body myositis: new insights and potential therapy. Curr Opin Neurol. 2014;27: 591-598.

20. Mastaglia FL, Needham M. Inclusion body myositis: a review of clinical and genetic aspects, diagnostic criteria and therapeutic approaches. J Clin Neurosci. 2015;22:6-13.

21. Needham M, Corbett A, Day T, Christiansen F, Fabian V, Mastaglia FL. Prevalence of sporadic inclusion body myositis and factors contributing to delayed diagnosis. J Clin Neurosci. 2008;15:1350-1353.
22. Cox FM, Titulaer MJ, SontJK, Wintzen AR, Verschuuren JJ, Badrising UA. A 12-year follow-up in sporadic inclusion body myositis: an end stage with major disabilities. Brain. 2011;134:3167-3175.

23. Eriksson M, Lindberg C. Hand function in 45 patients with sporadic inclusion body myositis. Occup Ther Int. 2012;19:108-116.

24. Benveniste O, Guiguet M, Freebody J, et al. Long-term observational study of sporadic inclusion body myositis. Brain. 2011;134:3176-3184.

25. Lindberg C, Oldfors A. Prognosis and prognostic factors in sporadic inclusion body myositis. Acta Neurol Scand. 2012;125:353-358.

26. Allenbach Y, Benveniste O, Decostre V, et al. Quadriceps strength is a sensitive marker of disease progression in sporadic inclusion body myositis. Neuromuscul Disord. 2012;22:980-986.

27. Hogrel JY, Allenbach Y, Canal A, et al. Four-year longitudinal study of clinical and functional endpoints in sporadic inclusion body myositis: implications for therapeutic trials. Neuromuscul Disord. 2014;24: 604-610.

28. Lowes LP, Alfano L, Viollet L, et al. Knee extensor strength exhibits potential to predict function in sporadic inclusion-body myositis. Muscle Nerve. 2012;45:163-168.

29. Rose MR, McDermott MP, Thornton CA, Palenski C, Martens WB, Griggs RC. A prospective natural history study of inclusion body myositis: implications for clinical trials. Neurology. 2001;57:548-550.

30. Jackson CE, Barohn RJ, Gronseth G, Pandya S, Herbelin L; Muscle Study Group. Inclusion body myositis functional rating scale: a reliable and valid measure of disease severity. Muscle Nerve. 2008;37: 473-476.

31. Lotz BP, Engel AG, Nishino H, Stevens JC, Litchy WJ. Inclusion body myositis. Observations in 40 patients. Brain. 1989;112(Pt 3):727-747.

32. Oh TH, Brumfield KA, Hoskin TL, Kasperbauer JL, Basford JR. Dysphagia in inclusion body myositis: clinical features, management, and clinical outcome. Am J Phys Med Rehabil. 2008;87:883-889.

33. Ko EH, Rubin AD. Dysphagia due to inclusion body myositis: case presentation and review of the literature. Ann Otol Rhinol Laryngol. 2014;123:605-608.

34. Ballo P, Chiodi L, Cameli M, et al. Dilated cardiomyopathy and inclusion body myositis. Neurol Sci. 2012;33:367-370.

35. Finsterer J, Stollberger C, Kovacs GG, Sehnal E. Left ventricular hypertrabeculation/noncompaction coincidentally found in sporadic inclusion body myositis. Int J Cardiol. 2013;168:610-612.

36. Inamori $Y$, Higuchi I, Inoue $T$, et al. Inclusion body myositis coexisting with hypertrophic cardiomyopathy: an autopsy study. Neuromuscul Disord. 2012;22:747-754.

37. Utz W, Schmidt S, Schulz-Menger J, Luft F, Spuler S. Cardiac involvement in sporadic inclusion-body myositis. Circulation. 2010;121: 706-708.

38. Della Marca G, Sancricca C, Losurdo A, et al. Sleep disordered breathing in a cohort of patients with sporadic inclusion body myositis. Clin Neurophysiol. 2013;124:1615-1621.

39. Rodriguez Cruz PM, Needham M, Hollingsworth P, Mastaglia FL, Hillman DR. Sleep disordered breathing and subclinical impairment of respiratory function are common in sporadic inclusion body myositis. Neuromuscul Disord. 2014;24:1036-1041.

40. Brady S, Squier W, Hilton-Jones D. Clinical assessment determines the diagnosis of inclusion body myositis independently of pathological features. J Neurol Neurosurg Psychiatry. 2013;84:1240-1246.

41. Sayers ME, Chou SM, Calabrese LH. Inclusion body myositis: analysis of 32 cases. J Rheumatol. 1992;19:1385-1389.

42. Lloyd TE, Mammen AL, Amato AA, Weiss MD, Needham M, Greenberg SA. Evaluation and construction of diagnostic criteria for inclusion body myositis. Neurology. 2014;83:426-433.

43. Chahin N, Engel AG. Correlation of muscle biopsy, clinical course, and outcome in PM and sporadic IBM. Neurology. 2008;70:418-424.

44. Girolamo F, Lia A, Amati A, et al. Overexpression of autophagic proteins in the skeletal muscle of sporadic inclusion body myositis. Neuropathol Appl Neurobiol. 2013;39:736-749.

45. Greenberg SA. Theories of the pathogenesis of inclusion body myositis. Curr Rheumatol Rep. 2010;12:221-228. 
46. Hiniker A, Daniels BH, Lee HS, Margeta M. Comparative utility of LC3, p62 and TDP-43 immunohistochemistry in differentiation of inclusion body myositis from polymyositis and related inflammatory myopathies. Acta Neuropathol Commun. 2013;1:29.

47. Weihl CC, Pestronk A. Sporadic inclusion body myositis: possible pathogenesis inferred from biomarkers. Curr Opin Neurol. 2010; 23:482-488.

48. Benveniste O, Stenzel W, Hilton-Jones D, Sandri M, Boyer O, van Engelen BG. Amyloid deposits and inflammatory infiltrates in sporadic inclusion body myositis: the inflammatory egg comes before the degenerative chicken. Acta Neuropathol. 2015;129:611-624.

49. De Bleecker JL, De Paepe B, Aronica E, et al. 205th ENMC International workshop: pathology diagnosis of idiopathic inflammatory myopathies part II 28-30 March 2014, Naarden, The Netherlands. Neuromuscul Disord. 2015;25:268-272.

50. Greenberg SA. Cytoplasmic 5'-nucleotidase autoantibodies in inclusion body myositis: isotypes and diagnostic utility. Muscle Nerve. 2014; 50:488-492.

51. Larman HB, Salajegheh M, Nazareno R, et al. Cytosolic 5'-nucleotidase 1A autoimmunity in sporadic inclusion body myositis. Ann Neurol. 2013; 73:408-418.

52. Lu X, Peng Q, Wang G. Discovery of new biomarkers of idiopathic inflammatory myopathy. Clin Chim Acta. 2015;444:117-125.

53. Pluk H, van Hoeve BJ, van Dooren SH, et al. Autoantibodies to cytosolic 5'-nucleotidase 1A in inclusion body myositis. Ann Neurol. 2013;73: 397-407.

54. Mastaglia FL. Sporadic inclusion body myositis: variability in prevalence and phenotype and influence of the MHC. Acta Myol. 2009;28: 66-71.

55. Mastaglia FL, Needham M, Scott A, et al. Sporadic inclusion body myositis: HLA-DRB1 allele interactions influence disease risk and clinical phenotype. Neuromuscul Disord. 2009;19:763-765.

56. Rojana-udomsart A, James I, Castley A, et al. High-resolution HLADRB1 genotyping in an Australian inclusion body myositis (s-IBM) cohort: an analysis of disease-associated alleles and diplotypes. J Neuroimmunol. 2012;250:77-82.

57. Scott AP, Laing NG, Mastaglia F, et al. Recombination mapping of the susceptibility region for sporadic inclusion body myositis within the major histocompatibility complex. J Neuroimmunol. 2011;235:77-83.

58. Rojana-udomsart A, Mitrpant C, James I, et al. Analysis of HLA-DRB3 alleles and supertypical genotypes in the MHC Class II region in sporadic inclusion body myositis. J Neuroimmunol. 2013;254:174-177.

59. Gang Q, Bettencourt C, Machado PM, et al. The effects of an intronic polymorphism in TOMM40 and APOE genotypes in sporadic inclusion body myositis. Neurobiol Aging. 2015;36:1766. e1-e3.

60. Tasca G, Monforte M, De Fino C, Kley RA, Ricci E, Mirabella M. MRI pattern recognition in sporadic inclusion body myositis. Muscle Nerve. Epub 25 March, 2015.

61. Dion E, Cherin P, Payan C, et al. Magnetic resonance imaging criteria for distinguishing between inclusion body myositis and polymyositis. J Rheumatol. 2002;29:1897-1906.

62. Phillips BA, Cala LA, Thickbroom GW, Melsom A, Zilko PJ, Mastaglia FL. Patterns of muscle involvement in inclusion body myositis: clinical and magnetic resonance imaging study. Muscle Nerve. 2001;24:1526-1534.

63. Cox FM, Reijnierse M, van Rijswijk CS, Wintzen AR, Verschuuren JJ, Badrising UA. Magnetic resonance imaging of skeletal muscles in sporadic inclusion body myositis. Rheumatology (Oxford). 2011;50: $1153-1161$.

64. Hiniker A, Daniels BH, Lee HS, Margeta M. Comparative utility of LC3, p62 and TDP-43 immunohistochemistry in differentiation of inclusion body myositis from polymyositis and related inflammatory myopathies. Acta Neuropathol Commun. 2013;1:29.

65. Arnardottir S, Alexanderson H, Lundberg IE, Borg K. Sporadic inclusion body myositis: pilot study on the effects of a home exercise program on muscle function, histopathology and inflammatory reaction. J Rehabil Med. 2003;35(1):31-35.
66. Barohn RJ, Amato AA, Sahenk Z, Kissel JT, Mendell JR. Inclusion body myositis: explanation for poor response to immunosuppressive therapy. Neurology. 1995;45:1302-1304.

67. Hengstman GJ, Ter Laak HJ, van Engelen BG, van Venrooij BG. AntiJo-1 positive inclusion body myositis with a marked and sustained clinical improvement after oral prednisone. J Neurol Neurosurg Psychiatry. 2001;70:706.

68. Lindberg C, Persson LI, Bjorkander J, Oldfors A. Inclusion body myositis: clinical, morphological, physiological and laboratory findings in 18 cases. Acta Neurol Scand. 1994;89:123-131.

69. Quartuccio L, De Marchi G, Scott CA, Ferraccioli G, Beltrami CA, De Vita S. Treatment of inclusion body myositis with cyclosporin-A or tacrolimus: successful long-term management in patients with earlier active disease and concomitant autoimmune features. Clin Exp Rheumatol. 2007;25:246-251.

70. Cherin P, Pelletier S, Teixeira A, et al. Intravenous immunoglobulin for dysphagia of inclusion body myositis. Neurology. 2002;58:326.

71. Dalakas MC, Sonies B, Dambrosia J, Sekul E, Cupler E, Sivakumar K. Treatment of inclusion-body myositis with IVIg: a double-blind, placebo-controlled study. Neurology. 1997;48:712-716.

72. Dobloug C, Walle-Hansen R, Gran JT, Molberg O. Long-term follow-up of sporadic inclusion body myositis treated with intravenous immunoglobulin: a retrospective study of 16 patients. Clin Exp Rheumatol. 2012;30:838-842.

73. Recher M, Sahrbacher U, Bremer J, Arndt B, Steiner U, Fontana A. Treatment of inclusion body myositis: is low-dose intravenous immunoglobulin the solution? Rheumatol Int. 2012;32:469-472.

74. Patwa HS, Chaudhry V, Katzberg H, Rae-Grant AD, So YT. Evidencebased guideline: intravenous immunoglobulin in the treatment of neuromuscular disorders: report of the Therapeutics and Technology Assessment Subcommittee of the American Academy of Neurology. Neurology. 2012;78:1009-1015.

75. Badrising UA, Maat-Schieman ML, Ferrari MD, et al. Comparison of weakness progression in inclusion body myositis during treatment with methotrexate or placebo. Ann Neurol. 2002;51:369-372.

76. Vordenbaumen S, Neuen-Jacob E, Richter J, Schneider M. Inclusion body myositis in a patient with long standing rheumatoid arthritis treated with anti-TNF $\alpha$ and rituximab. Clin Rheumatol. 2010;29:555-558.

77. Barohn RJ, Herbelin L, Kissel JT, et al. Pilot trial of etanercept in the treatment of inclusion-body myositis. Neurology. 2006;66:S123-S124.

78. Dastmalchi M, Grundtman C, Alexanderson H, et al. A high incidence of disease flares in an open pilot study of infliximab in patients with refractory inflammatory myopathies. Ann Rheum Dis. 2008;67: 1670-1677.

79. Dalakas MC, Rakocevic G, Schmidt J, et al. Effect of Alemtuzumab (CAMPATH 1-H) in patients with inclusion-body myositis. Brain. 2009;132:1536-1544.

80. Greenberg SA. Comment on alemtuzumab and inclusion body myositis. Brain. 2010;133:e135; author reply e6.

81. Terracciano C, Nogalska A, Engel WK, Askanas V. In AbetaPPoverexpressing cultured human muscle fibers proteasome inhibition enhances phosphorylation of AbetaPP751 and GSK3 $\beta$ activation: effects mitigated by lithium and apparently relevant to sporadic inclusion-body myositis. J Neurochem. 2010;112:389-396.

82. Kitazawa M, Trinh DN, LaFerla FM. Inflammation induces tau pathology in inclusion body myositis model via glycogen synthase kinase- $3 \beta$. Ann Neurol. 2008;64:15-24.

83. Dimachkie MM, Barohn RJ. Inclusion body myositis. Neurol Clin. 2014;32:629-646, vii.

84. Kosmidis ML, Alexopoulos H, Tzioufas AG, Dalakas MC. The effect of anakinra, an IL1 receptor antagonist, in patients with sporadic inclusion body myositis (sIBM): a small pilot study. J Neurol Sci. 2013;334: 123-125.

85. Zong M, Dorph C, Dastmalchi M, et al. Anakinra treatment in patients with refractory inflammatory myopathies and possible predictive response biomarkers: a mechanistic study with 12 months follow-up. Ann Rheum Dis. 2014;73:913-920. 
86. Morosetti R, Gliubizzi C, Broccolini A, Sancricca C, Mirabella M. Mesoangioblasts of inclusion-body myositis: a twofold tool to study pathogenic mechanisms and enhance defective muscle regeneration. Acta Myol. 2011;30:24-28.

87. Morosetti R, Mirabella M, Gliubizzi C, et al. MyoD expression restores defective myogenic differentiation of human mesoangioblasts from inclusion-body myositis muscle. Proc Natl Acad Sci US A. 2006;103: 16995-17000.

88. Northwestern University. Stem cell transplantation in idiopathic inflammatory myopathy diseases. Available from: https://clinicaltrials.gov/ ct2/show/NCT00278564. ClinicalTrials.gov identifier: NCT00278564. Accessed August 31, 2015.

89. Richard Barohn MD. Arimoclomol in sporadic inclusion body myositis. Available from: https://clinicaltrials.gov/ct2/show/NCT00769860. ClinicalTrials.gov identifier: NCT00769860. Accessed August 31, 2015.

90. Lahouti AH, Amato AA, Christopher-Stine L. Inclusion body myositis: update. Curr Opin Rheumatol. 2014;26:690-696.

91. Mendell JR, Rodino-Klapac L, Sahenk Z, et al. Gene therapy for muscular dystrophy: lessons learned and path forward. Neurosci Lett. 2012;527: 90-99.
92. Mendell JR, Sahenk Z, Malik V, et al. A phase $1 / 2$ a follistatin gene therapy trial for becker muscular dystrophy. Mol Ther. 2015;23:192-201.

93. Lach-Trifilieff E, Minetti GC, Sheppard K, et al. An antibody blocking activin type II receptors induces strong skeletal muscle hypertrophy and protects from atrophy. Mol Cell Biol. 2014;34:606-618.

94. Amato AA, Sivakumar K, Goyal N, et al. Treatment of sporadic inclusion body myositis with bimagrumab. Neurology. 2014;83:2239-2246.

95. Novartis Pharmaceuticals. Efficacy and safety of bimagrumab/BYM338 at 52 weeks on physical function, muscle strength, mobility in SIBM patients (RESILIENT). Available from: https://clinicaltrials.gov/ct2/ show/NCT01925209. ClinicalTrials.gov identifier: NCT01925209. Accessed August 31, 2015.

96. Alfano LN, Lowes LP, Dvorchik I, et al. The 2 min walk test is sufficient for evaluating walking abilities in sporadic inclusion body myositis. Neuromuscul Disord. 2014;24:222-226.
Therapeutics and Clinical Risk Management

\section{Publish your work in this journal}

Therapeutics and Clinical Risk Management is an international, peerreviewed journal of clinical therapeutics and risk management, focusing on concise rapid reporting of clinical studies in all therapeutic areas outcomes, safety, and programs for the effective, safe, and sustained use of medicines. This journal is indexed on PubMed Central, CAS,

\section{Dovepress}

EMBase, Scopus and the Elsevier Bibliographic databases. The manuscript management system is completely online and includes a very quick and fair peer-review system, which is all easy to use. Visit http://www.dovepress.com/testimonials.php to read real quotes from published authors.

Submit your manuscript here: http://www.dovepress.com/therapeutics-and-clinical-risk-management-journal 